\title{
Robustness of Adaptive Narrowband Beamforming With Respect to Bandwidth
}

\author{
Marc Oudin and Jean Pierre Delmas, Senior Member, IEEE
}

\begin{abstract}
This paper addresses the robustness of adaptive narrowband beamforming with respect to bandwidth based on the loss of performance in terms of signal-to-interference-plus-noise ratio (SINR). The criterion used by Zatman to define a narrowband environment, i.e., the ratio between the jammer plus noise covariance matrix and the noise eigenvalue, is studied from the point of view of a loss of SINR after narrowband beamforming under non narrowband conditions. Using theoretical results about the eigenvalues and eigenvectors of covariance matrices for signals closely spaced in frequency by Lee, it is shown that Zatman's criterion can be interpreted as an upper bound on the SINR loss which is nearly reached under certain conditions that are specified.
\end{abstract}

Index Terms-Adaptive beamforming, array signal processing, bandwidth, direction of arrival, narrowband, robustness, signal-tointerference-plus-noise ratio (SINR).

\section{INTRODUCTION}

$\mathbf{E}$ VOLUTION of radar technology and full digitized arrays allow the short term use of wideband waveforms for different advantages, such as high range resolution, stealthness, etc., (see, e.g., [1]). This breakthrough is a new challenge in radar for which broadband time domain or frequency domain array processing algorithms allow one to improve performance over standard spatial beamforming based on narrowband assumptions [2]. However, this improvement is done at the price of an increased complexity. Therefore, to optimize the choice between narrowband or broadband beamforming algorithms we have to precisely evaluate the performance of standard narrowband algorithms when the waveform has a certain given bandwidth.

In [3], Zatman proposes a general definition of a narrowband signal environment which is often used as a reference for adaptive beamforming, as well as for direction of arrival (DOA) estimation. It is based on the second eigenvalue of the jammer plus noise covariance matrix. Thus, an environment is qualified as "narrowband" if this eigenvalue is smaller than $3 \mathrm{~dB}$ above the noise level in the jammer plus noise covariance matrix. The author has shown by simulations that an increase of bandwidth leads to the second eigenvalue rising above the noise floor and a growth of the angular region in which the jammer denies coverage. Therefore, the decrease of performance of adap-

Manuscript received June 12, 2007; revised September 12, 2007. This work was supported in part by the French MOD (DGA) and in part by THALES/ TR6/SR. The associate editor coordinating the review of this manuscript and approving it for publication was Dr. Andreas Jakobsson.

The authors are with the Département CITI, GET/ Institut National des Télécommunications (INT), UMR-CNRS 5157, Institut National des Télécommunications, 91011 Evry Cedex, France (e-mail: marc.oudin@int-edu.eu; jeanpierre.delmas@int-edu.eu).

Color versions of one or more of the figures in this paper are available online at http://ieeexplore.ieee.org.

Digital Object Identifier 10.1109/TSP.2007.909358 tive beamforming algorithms is related to the second eigenvalue in the jammer plus noise covariance matrix. However, he has not given an explicit relation between the second eigenvalue level and the beamformer's performance losses. Moreover, he has considered a zero-bandwidth target whereas in practical applications, its bandwidth will often be nonzero like that of the jammer, which may also induce losses on the SINR. Those issues have been partially considered in [4] where the authors have proposed to define the ratio between the signal-to-interference-plus-noise ratio (SINR) resulting from narrowband beamforming with nonzero bandwidth conditions to that resulting from the same processing with zero bandwidth conditions, as a criterion for narrowband beamforming. However, they have considered a jammer-free environment, which is not realistic for most radar applications.

In this paper, we propose to use the same criterion as in [4] to study the robustness of adaptive narrowband beamforming, in the presence of a target and a nonzero bandwidth jammer whose DOAs are assumed to be arbitrary. First, we derive the expression of the SINR for zero-bandwidth and nonzero bandwidth target models. Using theoretical results about the eigenvalues and eigenvectors of covariance matrices for signals closely spaced in frequency by Lee [5], we show that under the assumption of a small fractional bandwidth, both models lead to the same expression. Then, we relate the considered SINR ratio to the criterion proposed by Zatman to define a narrowband environment, i.e., the ratio between the second eigenvalue of the jammer plus noise covariance matrix and the noise eigenvalue. Thus, we show that the latter criterion can be interpreted as an upper bound on the SINR loss due to bandwidth, with respect to the target DOA, and derive sufficient conditions for which the upper bound is nearly reached.

This paper is organized as follows. The data model is given in Section II. Then, the considered robustness criterion is given in Section III and detailed for zero-bandwidth and nonzero bandwidth target models. Finally, this criterion is related to Zatman's definition of narrowband in Section IV.

\section{Data Model}

We consider a radar system where the receive antenna is a uniform linear array ${ }^{1}$ (ULA) composed of $N$ sensors. The transmitted waveform has carrier frequency $f_{0}$ and the array is assumed to have half-wavelength spacing with respect to the carrier frequency. Then, consider an environment composed of one jammer, thermal noise and a target. The jammer is modeled by a nonzero bandwidth white stationary process with power $\sigma_{J}^{2}$ and bandwidth $B$ and the thermal noise by a spatially white complex

\footnotetext{
${ }^{1}$ The results of this paper are easily extended to an arbitrary array geometry.
} 
process, with power $\sigma_{n}^{2}$. The jamming plus noise covariance matrix ${ }^{2}$ is

$$
\overline{\mathbf{R}}=\int_{-\frac{B}{2}}^{\frac{B}{2}} \frac{\sigma_{J}^{2}}{B} \phi_{J}\left(f_{0}+f\right) \phi_{J}\left(f_{0}+f\right)^{H} d f+\sigma_{n}^{2} \mathbf{I}
$$

with

$$
\phi_{J}(f)=\left[\begin{array}{llll}
1 & e^{j \pi \frac{f}{f_{0}} u_{J}} & \ldots & e^{j(N-1) \pi \frac{f}{f_{0}} u_{J}}
\end{array}\right]^{T}
$$

where $u_{J}=\sin \left(\theta_{J}\right)$ and $\theta_{J}$ is the DOA of the jammer. Finally, the target signal is modelled by a stationary process, with power $\sigma_{S}^{2}$, known DOA $\theta_{S}$ and covariance matrix $\overline{\mathbf{R}}_{S}$.

\section{RobustNESS CRITERION WITH RESPECT TO BANDWIDTH}

The common performance measure of SINR is chosen to study the robustness of adaptive narrowband beamforming with respect to bandwidth. In practical cases, knowing whether a signal is narrowband or not is important to select the proper spatial processing. If the signal is narrowband, spatial processing alone is sufficient [6]. On the contrary, under non narrowband conditions, space-time or subband processing allows one to compensate for performance losses due to bandwidth, see, e.g., [2, ch. 6], [7]-[10].

Under nonzero bandwidth conditions, the SINR expression is given by

$$
\mathrm{SINR}=\frac{\mathbf{w}^{H} \overline{\mathbf{R}}_{S} \mathbf{w}}{\mathbf{w}^{H} \overline{\mathbf{R}} \mathbf{w}}
$$

where $\overline{\mathbf{R}}$ and $\overline{\mathbf{R}}_{S}$ are defined in the previous Section and $\mathbf{w}$ is a spatial filter. When a zero-bandwidth designed adaptive beamformer is computed under nonzero bandwidth conditions, its expression is given by ${ }^{3}$

$$
\mathbf{w}_{\mathrm{NZB}} \propto \overline{\mathbf{R}}^{-1} \phi_{S}
$$

and the SINR expression becomes, using (3) in (2)

$$
\operatorname{SINR}=\frac{\phi_{S}^{H} \overline{\mathbf{R}}^{-1} \overline{\mathbf{R}}_{S} \overline{\mathbf{R}}^{-1} \phi_{S}}{\phi_{S}^{H} \overline{\mathbf{R}}^{-1} \phi_{S}} .
$$

In practical applications, the target and jammer bandwidth can often be assumed to be identical. Indeed, when receiving a nonzero bandwidth target signal, a bandpass filter is often applied to the data, thus reducing the jammer bandwidth to that of the signal. Therefore, we consider in the following a nonzero bandwidth target with the same bandwidth $B$ as that of the jammer. However, to make the analysis of the full nonzero bandwidth case (i.e., nonzero bandwidth jammer and target) easier, we first consider the simpler case of a zero-bandwidth target model with a nonzero bandwidth jammer model. In the following, we detail SINR expression (4) depending on the target model.

\footnotetext{
${ }^{2} \mathrm{As}$ in [3], we consider a radar scenario, assuming that this covariance matrix does not contain a target signal's component and study steady-state performance.

${ }^{3}$ We use the subscript NZB when the environment is nonzero bandwidth and $\mathrm{ZB}$ when it is zero-bandwidth.
}

\section{A. Zero-Bandwidth Target Case}

Here, we assume that the target signal is modelled by a zerobandwidth stationary process. Its covariance matrix is given by

$$
\overline{\mathbf{R}}_{S} \stackrel{\text { def }}{=} \mathbf{R}_{S}=\sigma_{S}^{2} \phi_{S} \phi_{S}^{H}
$$

with

$$
\begin{aligned}
\phi_{S} & =\left[\begin{array}{llll}
1 & e^{j \pi u_{S}} & \ldots & e^{j(N-1) \pi u_{S}}
\end{array}\right]^{T} \\
\text { where } u_{S} & =\sin \left(\theta_{S}\right) .
\end{aligned}
$$

Injecting (5) in (4), we obtain

$$
\operatorname{SINR}_{\mathrm{NZB}}=\sigma_{S}^{2} \phi_{S}^{H} \overline{\mathbf{R}}^{-1} \phi_{S} .
$$

\section{B. Nonzero Bandwidth Target Case}

Here, we assume that the target is modelled by a nonzero bandwidth white stationary process with bandwidth $B$. Its covariance matrix may be written as ${ }^{4}$

$$
\overline{\mathbf{R}}_{S}=\int_{-\frac{B}{2}}^{\frac{B}{2}} \frac{\sigma_{S}^{2}}{B} \phi_{S}\left(f_{0}+f\right) \phi_{S}\left(f_{0}+f\right)^{H} d f
$$

with

$$
\phi_{S}(f)=\left[\begin{array}{llll}
1 & e^{j \pi \frac{f}{f_{0}} u_{S}} & \ldots & e^{j(N-1) \pi \frac{f}{f_{0}} u_{S}}
\end{array}\right]^{T} .
$$

Now we show that (4) can be approximated by the SINR expression obtained with a zero-bandwidth target (7) under the assumption of a small fractional bandwidth. More precisely, we prove in Appendix I the following result.

Result 1: When the second eigenvalue of the target covariance matrix $\lambda_{2}$ satisfies $\lambda_{2} \ll\left(\left(\sigma_{n}^{2}\right) /\left(\sigma_{J}^{2}\right)\right) \sigma_{S}^{2}$, the SINR appearing in criterion (4) is approximated by

$$
\operatorname{SINR}_{\mathrm{NZB}}=\sigma_{S}^{2} \phi_{S}^{H} \overline{\mathbf{R}}^{-1} \phi_{S} .
$$

This approximation is validated by extensive numerical comparisons, for arbitrary jammer DOAs and number of sensors. For instance, Fig. 1 compares actual SINR (4) to approximate one (9) as a function of the target DOA. The parameters are $N=10, \sigma_{J}^{2}=30, \sigma_{S}^{2}=0$, and $\sigma_{n}^{2}=0 \mathrm{~dB}, u_{J}=0.1$ (those parameters will be used in the following simulations) and the fractional bandwidth $B / f_{0}=0.15$. We observe that the approximation is accurate except when the target DOA is close to array endfire. Then, to illustrate the influence of the fractional bandwidth, we plot in Fig. 2 the same expressions, as well as the term $\rho=\left(\lambda_{2} \sigma_{J}^{2}\right) /\left(\sigma_{n}^{2} \sigma_{S}^{2}\right)$, as a function of the fractional bandwidth, with $u_{S}=0.15$. First, we note that the approximation remains accurate for fractional bandwidths up to about 0.15 . Then, we observe that $\lambda_{2}$ rapidly increases with the SINR and that the condition in Result 1 is sufficient but not necessary. Indeed, the approximation may be very accurate whereas the condition $\left(\lambda_{2} \sigma_{J}^{2}\right) /\left(\sigma_{n}^{2} \sigma_{S}^{2}\right) \ll 1$ is not satisfied, due to the coarse upper-bounds used for the derivation of Result 1.

\footnotetext{
${ }^{4}$ Note that in [4], a simpler rank-one target covariance matrix of the form $\overline{\mathbf{R}}_{S}=\sigma_{S}^{2} \bar{\phi}_{S} \bar{\phi}_{S}^{H}$ with $\bar{\phi}_{S} \propto \int_{-B / 2}^{B / 2} \phi_{S}\left(f_{0}+f\right) d f$ was used.
} 


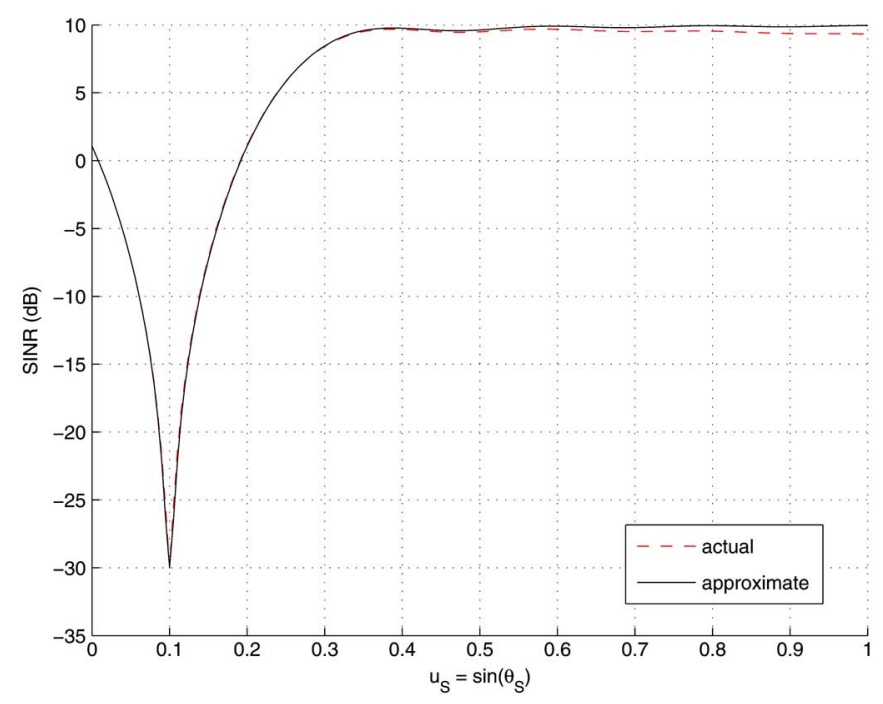

Fig. 1. Actual SINR (4) and approximate SINR (9), as a function of the target DOA.

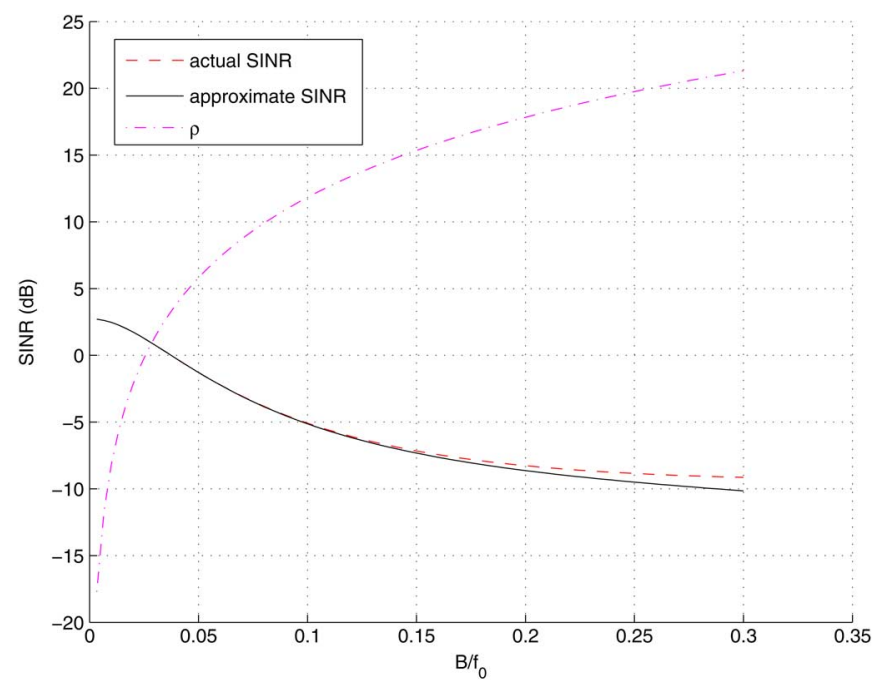

Fig. 2. Actual SINR (4), approximate SINR (9) and $\rho=\left(\lambda_{2} \sigma_{J}^{2}\right) /\left(\sigma_{n}^{2} \sigma_{S}^{2}\right)$ as a function of the fractional bandwidth.

\section{Expression of the Robustness Criterion}

Here, we introduce a robustness criterion defined by the ratio between the nonzero bandwidth SINR (for both zero-bandwidth and nonzero bandwidth targets with $\left.\left(B / f_{0}\right) \ll 1\right)$ and the zero-bandwidth SINR [4]. This criterion allows one to quantify the loss in SINR due to the increase of bandwidth of the environment (see, e.g., [3] for illustrations of this loss in SINR). Its expression is

$$
r=\frac{\operatorname{SINR}_{\mathrm{NZB}}}{\mathrm{SINR}_{\mathrm{ZB}}}
$$

where SINR $_{N Z B}$ is given by (7) and (9) and SINR ${ }_{Z B}$ is the optimal SINR under zero-bandwidth conditions equal to

$$
\mathrm{SINR}_{\mathrm{ZB}}=\sigma_{S}^{2} \phi_{S}^{H} \mathbf{R}^{-1} \phi_{S}
$$

where

$$
\mathbf{R}=\sigma_{J}^{2} \phi_{J} \phi_{J}^{H}+\sigma_{n}^{2} \mathbf{I}
$$

is the zero-bandwidth jamming plus noise covariance matrix, and $\phi_{J}=\phi_{J}\left(f_{0}\right)$ is the zero-bandwidth jammer steering vector. Because $r<1, r^{-1}$ will denote the SINR loss throughout the paper. Using the expressions of $\mathrm{SINR}_{\mathrm{NZB}}$ and $\mathrm{SINR}_{\mathrm{ZB}}$ in (10), we obtain the detailed form of the chosen robustness criterion

$$
r=\frac{\phi_{S}^{H} \overline{\mathbf{R}}^{-1} \phi_{S}}{\phi_{S}^{H} \mathbf{R}^{-1} \phi_{S}} .
$$

\section{RELATION BETWEEN THE SINR LOSS AND ZATMAN'S DEFINITION OF NARROWBAND}

Now, we want to relate SINR ratio (12) to the ratio between the second eigenvalue of the jammer plus noise covariance matrix and the noise eigenvalue, proposed by Zatman for defining a narrowband environment.

\section{A. Upper Bound on the SINR Loss}

Assuming that the fractional bandwidth is small, we prove in the conditions of Zatman [3] the following result:

Result 2: In the presence of a zero-bandwidth target and a nonzero bandwidth jammer, the ratio between the second eigenvalue of the jammer plus noise covariance matrix and the noise eigenvalue is an upper bound on the SINR loss $r^{-1}$ of the optimal adaptive beamformer due to bandwidth with respect to the target DOA.

Proof: Using the derivation given in Appendix I and based on the results of [5], the nonzero bandwidth jammer covariance matrix $\overline{\mathbf{R}}-\sigma_{n}^{2} \mathbf{I}$ can be approximated by a rank-two matrix ${ }^{5}$ where its largest two eigenvalues and the associated eigenvector are, respectively, $\mu_{1}-\sigma_{n}^{2} \approx N \sigma_{J}^{2}$, $\mu_{2}-\sigma_{n}^{2}$ and $\mathbf{u}_{1} \approx \phi_{J} / \sqrt{N}$ and $\mathbf{u}_{2}$. Then, using (11), $\mathbf{R}-\sigma_{n}^{2} \mathbf{I}=N \sigma_{J}^{2}\left(\phi_{J} / \sqrt{N}\right)\left(\phi_{J}^{H} / \sqrt{N}\right)$. Consequently, we have

$$
\overline{\mathbf{R}} \approx \mathbf{R}+\left(\mu_{2}-\sigma_{n}^{2}\right) \mathbf{u}_{2} \mathbf{u}_{2}^{H} .
$$

Using the matrix inversion lemma, we obtain

$\overline{\mathbf{R}}^{-1} \approx \mathbf{R}^{-1}-\mathbf{R}^{-1} \mathbf{u}_{2}\left(\frac{1}{\mu_{2}-\sigma_{n}^{2}}+\mathbf{u}_{2}^{H} \mathbf{R}^{-1} \mathbf{u}_{2}\right)^{-1} \mathbf{u}_{2}^{H} \mathbf{R}^{-1}$.

Incorporating (13) in (12), we obtain

$$
r \approx 1-\frac{\left|\phi_{S}^{H} \mathbf{R}^{-1} \mathbf{u}_{2}\right|^{2}}{\phi_{S}^{H} \mathbf{R}^{-1} \phi_{S}} \frac{1}{\left(\frac{1}{\mu_{2}-\sigma_{n}^{2}}+\mathbf{u}_{2}^{H} \mathbf{R}^{-1} \mathbf{u}_{2}\right)} .
$$

A lower bound of this SINR ratio (14) with respect to the target DOA is obtained by considering unconstrained steering vector $\phi_{S}$. In this case, (14) is minimized when the term $\left(\left|\phi_{S}^{H} \mathbf{R}^{-1} \mathbf{u}_{2}\right|^{2}\right) /\left(\phi_{S}^{H} \mathbf{R}^{-1} \phi_{S}\right)$ is maximized, i.e.,

${ }^{5}$ Note that this assumption has been justified in [3] by the empirical observation that the eigenvalues of $\overline{\mathbf{R}}$ overtake the noise floor one at a time when the bandwidth is increased. 
with $\phi_{S} \propto \mathbf{u}_{2}$. Using, $\mathbf{u}_{2}^{H} \mathbf{R}^{-1} \mathbf{u}_{2} \approx\left(1 / \sigma_{n}^{2}\right)$ derived from $\mathbf{u}_{2}^{H}\left(\phi_{J} / \sqrt{N}\right) \approx \mathbf{u}_{2}^{H} \mathbf{u}_{1}=0$, this associated lower bound on the SINR ratio $r$ is equal to 6

$$
r_{l b}=\frac{\sigma_{n}^{2}}{\mu_{2}}
$$

Consequently, the ratio between the noise eigenvalue and the second eigenvalue of the jammer plus noise covariance matrix can be interpreted as a lower bound on the SINR ratio $r$ or conversely, the ratio between the second eigenvalue of the jammer plus noise covariance matrix and the noise eigenvalue as an upper bound on the SINR loss. From this result, we deduce an upper bound of the SINR loss $r^{-1}$ in the presence of a narrowband jammer, in the sense of Zatman's definition. Thus, when the second eigenvalue of the jammer plus noise covariance matrix is smaller than $3 \mathrm{~dB}$ above the noise eigenvalue, Result 2 proves that the SINR loss will be smaller than $3 \mathrm{~dB}$ for arbitrary target and jammer DOAs. Indeed, if $\mu_{2} \leq 2 \sigma_{n}^{2}$, we have

$$
1 \leq \text { SINR loss }=\frac{1}{r} \leq \frac{1}{r_{l b}}=\frac{\mu_{2}}{\sigma_{n}^{2}} \leq 2 .
$$

For a nonzero bandwidth target, we have shown in Section III that the SINR expression could be precisely approximated by the SINR expression in the presence of a zero-bandwidth target, under the assumption that $\lambda_{2} \ll\left(\left(\sigma_{n}^{2}\right) /\left(\sigma_{J}^{2}\right)\right) \sigma_{S}^{2}$ where $\lambda_{2}$ is the second eigenvalue of the target covariance matrix (see Result 1). Therefore, Result 2 is also valid for a nonzero bandwidth target, under the latter assumption.

After having given a general relation between Zatman's criterion and the SINR loss $r^{-1}$, we now want to give sufficient conditions for which the upper bound $r_{l b}^{-1}$ is nearly reached for a certain DOA of the target.

\section{B. Derivation of Sufficient Conditions for Which the Upper Bound Is Nearly Reached}

For ease of notations, we only consider the zero-bandwidth target model. However, as we have already noted, the analysis remains valid in the presence of a nonzero bandwidth target under the conditions given by Result 1 .

Our aim is to analyze the SINR ratio given by (12). To proceed, we first use the approximation introduced in [3] and justified by the analysis of Lee [5] applied to the spectral representation of stationary bandlimited signals (see Appendix I), to replace the jammer covariance by a rank-two matrix, under the assumption of a small fractional bandwidth. Then, we make the second assumption that the array is composed of many sensors 7 which allows one to derive limit expressions of the considered SINR ratio.

${ }^{6}$ Note that an approximation of $\mathbf{u}_{2}$ is given by the derivative of $\phi_{J}(f)$ with respect to $f$, orthogonalized by $\phi_{J}, \mathbf{u}_{2} \approx$ $\left(\left(\mathbf{I} \quad-\quad\left(\phi_{J} \phi_{J}^{H}\right) /(N)\left(d \phi_{J}(f) / d f\right)\right\rfloor_{f=f_{0}}\right) /\left(\| \mathbf{I} \quad-\quad\left(\phi_{J} \phi_{J}^{H}\right) /\right.$ $\left.\left.(N)\left(d \phi_{J}(f)\right) /(d f)\right\rfloor_{f=f_{0}} \|\right)+o\left((B) /\left(f_{0}\right)\right)$ [5]. Since there is no target DOA for which $\phi_{S}$ is proportional to this vector, the lower bound on the SINR ratio can not be reached.

${ }^{7}$ This assumption is justified in most radar applications, for which high spatial resolution is required.

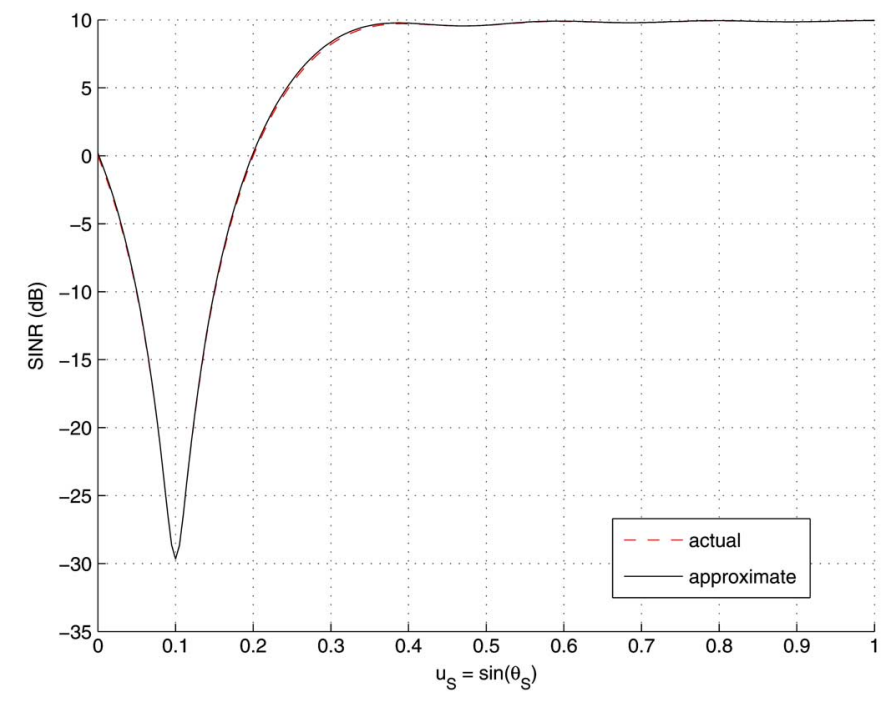

Fig. 3. Actual SINR (7) with or without approximation of the jammer plus noise covariance matrix by (16) as a function of the target DOA.

1) Approximation of Covariance Matrix: We use the following approximation:

$$
\tilde{\mathbf{R}}=\frac{\sigma_{J}^{2} \phi_{J, 1} \phi_{J, 1}^{H}+\sigma_{J}^{2} \phi_{J, 2} \phi_{J, 2}^{H}}{2}+\sigma_{n}^{2} \mathbf{I}
$$

with

$$
\begin{aligned}
& \phi_{J, 1}=\phi_{J}\left(f_{0}-\Delta f\right) \\
& \phi_{J, 2}=\phi_{J}\left(f_{0}+\Delta f\right)
\end{aligned}
$$

where $\Delta f=(B) /(2 \sqrt{3})$. We have validated this second approximation by extensive numerical comparisons. However, we note that the rank-two approximation leads to significant errors in the SINR expression, when the fractional bandwidth is too large. Indeed, in that case, the effective rank of the covariance matrix is larger than 2 and the approximation is not justified anymore. However, simulations show that for fractional bandwidths up to $(B) /\left(f_{0}\right)=0.3$, with the chosen parameters, this approximation remains acceptable. It is illustrated in Fig. 3 where we plot expression (7) with or without approximation of the covariance matrix according to (16) for $B / f_{0}=0.3$ as a function of the target DOA and in Fig. 4 for $u_{S}=0.15$ as a function of the fractional bandwidth.

We observe that errors due to this second approximation are very small. Therefore, (16) may be used for analysis of the nonzero bandwidth case at small fractional bandwidths.

2) Derivation of Limit Expressions of the SINR Ratio: The approximation of $\overline{\mathbf{R}}$ in (12) by $\tilde{\mathbf{R}}$ allows one to derive a closedform expression of $\operatorname{SINR}_{\mathrm{NZB}}$ (7) and then of $r$. Indeed, after a double application of the matrix inversion lemma, we can write

$$
\begin{aligned}
\phi_{S}^{H} \tilde{\mathbf{R}}^{-1} \phi_{S}= & \frac{N}{\sigma_{n}^{2}}-\frac{\left|\phi_{S}^{H} \phi_{J, 2}\right|^{2}}{\sigma_{n}^{4} \beta}-\frac{\left|\phi_{S}^{H} \phi_{J, 1}\right|^{2}}{\sigma_{n}^{4} \alpha} \\
& -\frac{\left|\phi_{S}^{H} \phi_{J, 2}\right|^{2}\left|\phi_{J, 2}^{H} \phi_{J, 1}\right|^{2}}{\sigma_{n}^{8} \beta^{2} \alpha} \\
& +\frac{2}{\sigma_{n}^{6} \beta \alpha} \operatorname{Re}\left[\left(\phi_{S}^{H} \phi_{J, 2}\right)\left(\phi_{J, 1}^{H} \phi_{S}\right)\left(\phi_{J, 2}^{H} \phi_{J, 1}\right)\right]
\end{aligned}
$$




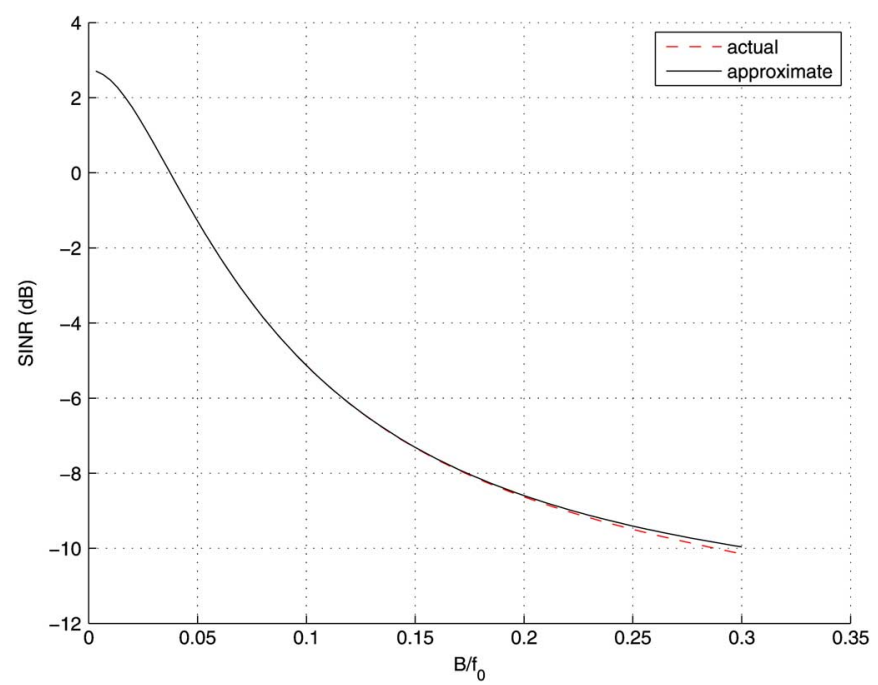

Fig. 4. Actual SINR (7) with or without approximation of the jammer plus noise covariance matrix by (16) as a function of the fractional bandwidth.

with $\beta=(2) /\left(\sigma_{J}^{2}\right)+(N) /\left(\sigma_{n}^{2}\right), \alpha=\beta-\left(\left|\phi_{J, 1}^{H} \phi_{J, 2}\right|^{2}\right) /\left(\sigma_{n}^{4} \beta\right)$ and

$$
\begin{aligned}
\phi_{S}^{H} \phi_{J, 1} & =e^{-j(N-1) x_{1}} \frac{\sin \left(N x_{1}\right)}{\sin \left(x_{1}\right)} \\
\phi_{S}^{H} \phi_{J, 2} & =e^{-j(N-1) x_{2}} \frac{\sin \left(N x_{2}\right)}{\sin \left(x_{2}\right)} \\
\phi_{J, 2}^{H} \phi_{J, 1} & =e^{-j 2(N-1) \Delta x} \frac{\sin (2 N \Delta x)}{\sin (2 \Delta x)}
\end{aligned}
$$

where

$$
\begin{aligned}
x_{1} & =x_{0}+\Delta x \\
x_{2} & =x_{0}-\Delta x \\
x_{0} & =\frac{\pi}{2}\left(u_{S}-u_{J}\right) \\
\Delta x & =\frac{\pi}{2} \frac{\Delta f}{f_{0}} u_{J} .
\end{aligned}
$$

Then after a Taylor series expansion, using $N \gg 1$ and $(\Delta f) /\left(f_{0}\right) \ll 1$ under constraint $N(\Delta f) /\left(f_{0}\right) \ll 1$, and $\left(\sigma_{J}^{2}\right) /\left(\sigma_{n}^{2}\right) \gg 1$, we can write for $x_{0} \neq 0$

$$
\phi_{S}^{H} \tilde{\mathbf{R}}^{-1} \phi_{S} \approx \phi_{S}^{H} \mathbf{R}^{-1} \phi_{S}-\frac{b^{2}}{4 a}\left(\frac{3 \sigma_{J}^{2} \Delta x^{2}}{N^{3} \sigma_{n}^{2} \sigma_{J}^{2} \Delta x^{2}+3 \sigma_{n}^{4}}\right)
$$

with

$$
\left\{\begin{array}{l}
a=\frac{\sin ^{2}\left(N x_{0}\right)}{\sin ^{2}\left(x_{0}\right)} \\
b=\frac{N \sin ^{2}\left(2 N x_{0}\right)}{\sin ^{2}\left(x_{0}\right)}-\frac{\sin ^{2}\left(N x_{0}\right) \sin \left(2 x_{0}\right)}{\sin ^{4}\left(x_{0}\right)} .
\end{array}\right.
$$

We deduce an approximate expression of the proposed criterion

$$
r \approx 1-\frac{\frac{b^{2}}{4 a}\left(\frac{3 \sigma_{J}^{2} \Delta x^{2}}{N^{3} \sigma_{n}^{2} \sigma_{J}^{2} \Delta x^{2}+3 \sigma_{n}^{4}}\right)}{\phi_{S}^{H} \mathbf{R}^{-1} \phi_{S}} .
$$

In order to validate the latter equation, we now compare in Fig. 5 approximate relation (19) with actual one (12)

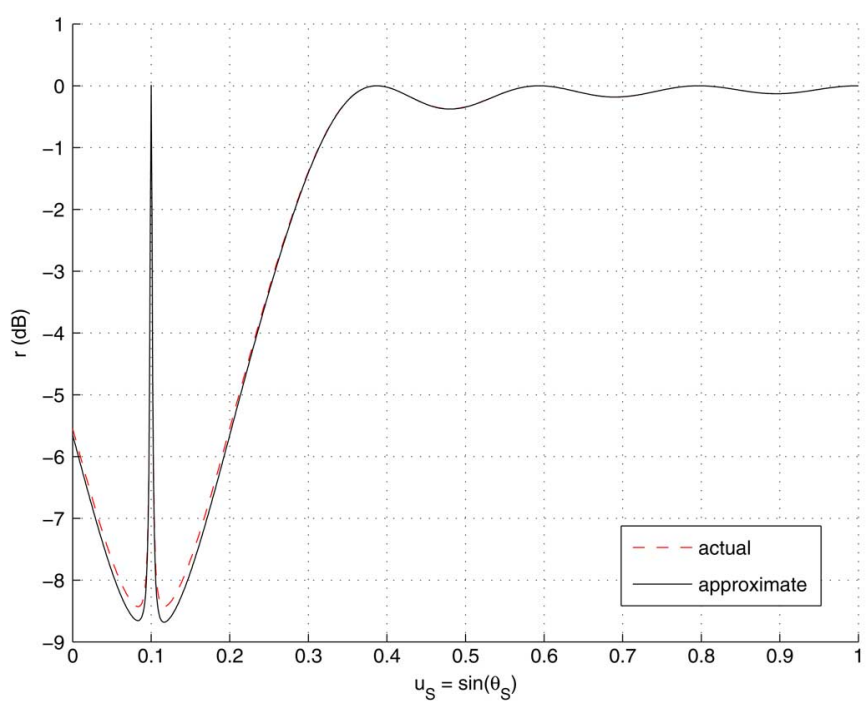

Fig. 5. Actual SINR ratio (12) and approximate SINR ratio (19), as a function of the target DOA.

with respect to the target DOA. The fractional bandwidth is $(B) /\left(f_{0}\right)=0.1$.

First, we observe that the approximate plot is an accurate estimate of $r$. We notice that when the target is in the vicinity of the jammer, the SINR losses $r^{-1}$ increase until a target position close to that of the jammer. Then, from that position to the jammer one, the SINR losses quickly decrease. When the target and jammer DOAs are equal, the losses have nearly vanished (actual ratio (12) is equal to $\left(\sigma_{n}^{2}+N \sigma_{J}^{2}\right) /\left(\mu_{2}\right) \approx 1$ [5] where $\mu_{1}$ is the largest eigenvalue of $\overline{\mathbf{R}}$ ).

Next, we want to estimate the "worst-case" SINR ratio $r_{\min }$ with respect to the target DOA and relate it to the lower bound $r_{l b}$ (15). We prove the following result:

Result 3: Under the assumptions that $\left(B / f_{0}\right) \ll 1$ and $N \gg$ 1 under constraint $N\left(B / f_{0}\right) \ll 1$, the maximal SINR loss $r_{\min }^{-1}$ with respect to the target DOA nearly reaches the upper bound $r_{l b}^{-1}$ equal to the ratio between the second eigenvalue of the jammer plus noise covariance matrix and the noise eigenvalue.

Proof: Noticing that the minimum SINR ratio $r_{\min }$ is reached when $0<\left|x_{0}\right| \ll 1$, we can use a Taylor series expansion of term $\left(b^{2} / a\right)$ in (19), and obtain after a few algebraic manipulations summarized in Appendix II

$$
\frac{b^{2}}{a} \approx \frac{4}{9} N^{6} x_{0}^{2} .
$$

Then, by noticing that $\phi_{S}^{H} \mathbf{R}^{-1} \phi_{S} \approx\left(N^{3} x_{0}^{2}\right) /\left(3 \sigma_{n}^{2}\right)$ for $\left(\sigma_{J}^{2}\right) /\left(\sigma_{n}^{2}\right) \gg 1,0<\left|x_{0}\right| \ll 1$ and using (17), we obtain the following approximation of the minimum value of $r$ :

$$
\begin{aligned}
r_{\text {min }} & \approx \frac{\sigma_{n}^{2}}{\frac{N^{3}}{3} \sigma_{J}^{2} \Delta x^{2}+\sigma_{n}^{2}} \\
& =\frac{\sigma_{n}^{2}}{\frac{N^{3}}{3} \sigma_{J}^{2} \frac{\pi^{2}}{48}\left(\frac{B}{f_{0}}\right)^{2} u_{J}^{2}+\sigma_{n}^{2}} .
\end{aligned}
$$

We remark that $\left(N^{3} / 3\right) \sigma_{J}^{2}\left(\pi^{2} / 48\right)\left(B / f_{0}\right)^{2} u_{J}^{2}$ represents the first-order Taylor series expansion of the second eigenvalue of the jammer's noise-free covariance matrix [deduced from ([3, 


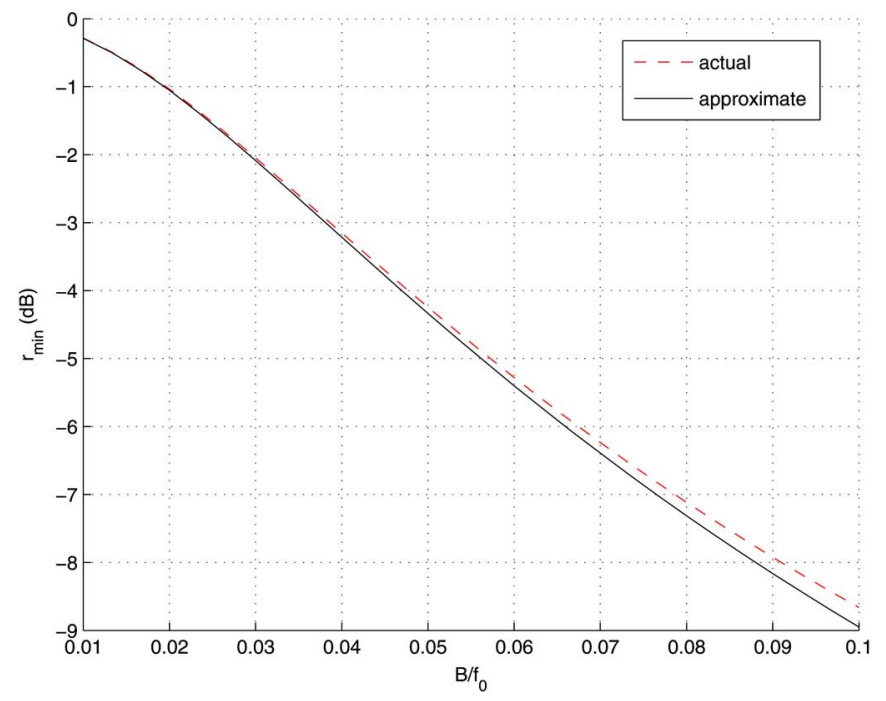

Fig. 6. Actual and approximate (21) values of $r_{\min }$, as a function of the fractional bandwidth.

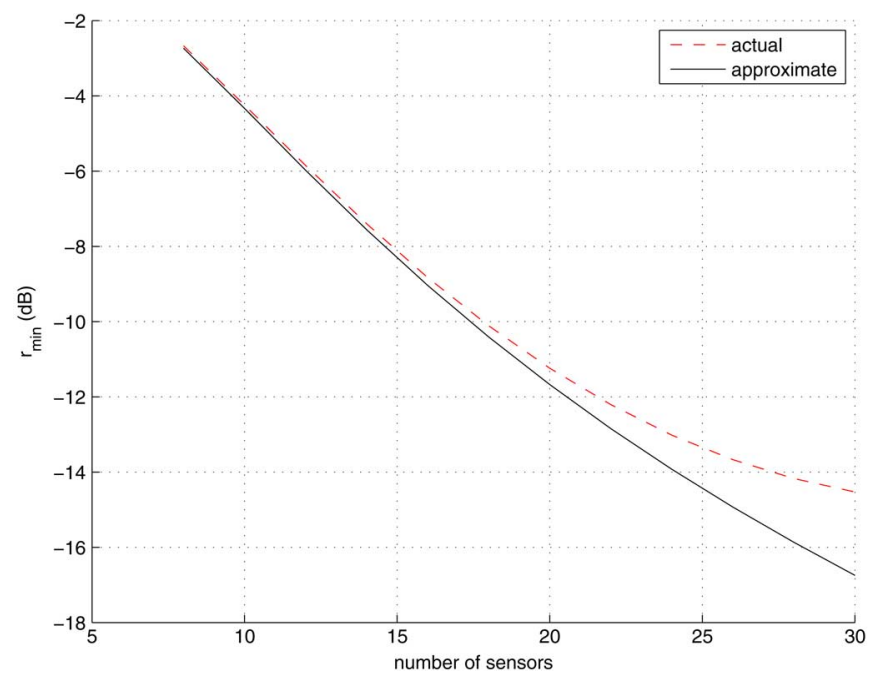

Fig. 7. Actual and approximate (21) values of $r_{\min }$, as a function of the number of sensors.

rels 27 and 28]) for $(N B) /\left(f_{0}\right) \ll 1$ ], so that the denominator of (21) approximates $\mu_{2}$. Finally, taking the inverse of (21) completes the proof.

In order to observe the influence of the fractional bandwidth with respect to minimal SINR ratio $r_{\min }$, we now plot in Fig. 6 this one as a function of $\left(B / f_{0}\right)$.

First, we note that approximate expression (21) is a very accurate estimate of the actual value $r_{\min }$ obtained from (12), except for important fractional bandwidths. Second, we observe that $r_{\min }$ rapidly decreases when the fractional bandwidth increases. Then, we analyze the influence of the number of sensors on $r_{\min }$. Fig. 7 shows the value of this criterion for the actual and approximate expressions, for different values of $N$ and $\left(B / f_{0}\right)=0.05$.

We observe that the approximation given by (21) is very accurate, except for high values of $N$. This can be explained by the fact that the series expansion done previously is valid under the hypothesis that $N(\Delta f) /\left(f_{0}\right) \ll 1$.

\section{CONCLUSION}

In this paper, the robustness of adaptive narrowband beamforming with respect to bandwidth has been studied where the criterion of the loss of performance of the standard narrowband processing in terms of SINR, under the assumption of a nonzero bandwidth environment, with respect to the narrowband case has been proposed. Using results about the eigenvalues and eigenvectors of the covariance matrix for signals closely spaced in frequency, this SINR loss has been related to the ratio between the second eigenvalue of the jammer plus noise covariance matrix and the noise eigenvalue. Thus, it has been shown that under the assumption of a small fractional bandwidth, the SINR loss is upper bounded by the ratio between the second eigenvalue of the jammer plus noise covariance matrix and the noise eigenvalue, for both zero-bandwidth and nonzero bandwidth target models. Then, sufficient conditions for which the upper bound of the SINR loss is nearly reached have been given.

\section{APPENDIX I \\ PROOF OF RESULT 1}

First, consider the EVD of $\overline{\mathbf{R}}_{S}$

$$
\overline{\mathbf{R}}_{S}=\sum_{n=1}^{N} \lambda_{n} \mathbf{v}_{n} \mathbf{v}_{n}^{H} .
$$

Using the spectral representation $x_{t}=\int_{-B / 2}^{B / 2} e^{j 2 \pi f t} d \mu(f)$ of the complex envelope of the wide-sense stationary bandlimited Gaussian target signals, approximated [11] by $x_{t} \approx \sum_{l=0}^{L-1} a_{l} e^{j 2 \pi f_{l} t}$ with $f_{l}=(-L+2 l+1)(B / 2 L), L \gg 1$ and $\left(a_{l}\right)_{l=0, \ldots, L-1}$ uncorrelated Gaussian random variables with $E\left\{\left|a_{l}\right|^{2}\right\}=\left(\sigma_{J}^{2}\right) /(L), \overline{\mathbf{R}}_{S}$ can be approximated as the spatial covariance associated with a discrete sum of zero-bandwidth signals closely spaced in frequency for low fractional bandwidths

$$
\overline{\mathbf{R}}_{S} \approx \sum_{l=0}^{L-1} \frac{\sigma_{S}^{2}}{L} \phi_{S}\left(f_{0}+f_{l}\right) \phi_{S}^{H}\left(f_{0}+f_{l}\right) .
$$

Consequently, the results of [5] apply. In particular

$$
\begin{aligned}
& \mathbf{v}_{1}=\frac{\phi_{S}}{\sqrt{N}}+O\left(\frac{B}{f_{0}}\right) \\
& \text { and } \\
& \lambda_{1}=\operatorname{tr}\left(\overline{\mathbf{R}}_{S}\right)+O\left(\frac{B}{f_{0}}\right), \\
& \lambda_{n}=O\left[\left(\frac{B}{f_{0}}\right)^{2(n-1)}\right] \text { for } n>1
\end{aligned}
$$

Therefore, for $B / f_{0} \ll 1, \mathbf{v}_{1} \approx\left(\phi_{S}\right) /(\sqrt{N})$ and $\lambda_{1} \approx N \sigma_{S}^{2}$ and the nonzero bandwidth target covariance matrix $\overline{\mathbf{R}}_{S}$ can be approximated by the rank-two matrix

$$
\overline{\mathbf{R}}_{S} \approx \sigma_{S}^{2} \phi_{S} \phi_{S}^{H}+\lambda_{2} \mathbf{v}_{2} \mathbf{v}_{2}^{H} .
$$

Injecting (22) in (4), we obtain

$$
\operatorname{SINR} \approx \sigma_{S}^{2} \phi_{S}^{H} \overline{\mathbf{R}}^{-1} \phi_{S}\left(1+\frac{\lambda_{2}\left|\phi_{S}^{H} \overline{\mathbf{R}}^{-1} \mathbf{v}_{2}\right|^{2}}{\sigma_{S}^{2}\left(\phi_{S}^{H} \overline{\mathbf{R}}^{-1} \phi_{S}\right)^{2}}\right) .
$$


Now, we derive a sufficient condition to neglect the second term in (23). To proceed, let consider the following ratio:

$$
\frac{\lambda_{2}\left|\phi_{S}^{H} \overline{\mathbf{R}}^{-1} \mathbf{v}_{2}\right|^{2}}{\sigma_{S}^{2}\left(\phi_{S}^{H} \overline{\mathbf{R}}^{-1} \phi_{S}\right)^{2}}
$$

and compare it to unity. Using the Cauchy-Schwartz inequality, we have

$$
\frac{\lambda_{2}\left|\phi_{S}^{H} \overline{\mathbf{R}}^{-1} \mathbf{v}_{2}\right|^{2}}{\sigma_{S}^{2}\left(\phi_{S}^{H} \overline{\mathbf{R}}^{-1} \phi_{S}\right)^{2}} \leq \frac{\lambda_{2} \mathbf{v}_{2}^{H} \overline{\mathbf{R}}^{-1} \mathbf{v}_{2}}{\sigma_{S}^{2} \phi_{S}^{H} \overline{\mathbf{R}}^{-1} \phi_{S}} .
$$

Then, since $\mathbf{v}_{2}^{H} \overline{\mathbf{R}}^{-1} \mathbf{v}_{2} \leq 1 / \mu_{N} \leq 1 / \sigma_{n}^{2}$ and $\phi_{S}^{H} \overline{\mathbf{R}}^{-1} \phi_{S} \geq$ $N / \mu_{1}$ where $\mu_{1}$ and $\mu_{N}$ are given by the EVD of $\overline{\mathbf{R}}=$ $\sum_{n=1}^{N} \mu_{n} \mathbf{u}_{n} \mathbf{u}_{n}^{H}$, we obtain

$$
\frac{\lambda_{2}\left|\phi_{S}^{H} \overline{\mathbf{R}}^{-1} \mathbf{v}_{2}\right|^{2}}{\sigma_{S}^{2}\left(\phi_{S}^{H} \overline{\mathbf{R}}^{-1} \phi_{S}\right)^{2}} \leq \frac{\lambda_{2} \mu_{1}}{N \sigma_{S}^{2} \sigma_{n}^{2}} .
$$

A sufficient condition to neglect the second term in (9) is, therefore, that

$$
\frac{\lambda_{2} \mu_{1}}{N \sigma_{S}^{2} \sigma_{n}^{2}} \ll 1
$$

and since $\mu_{1} \approx N \sigma_{J}^{2}$ for $\left(B / f_{0}\right) \ll 1$, this condition becomes

$$
\lambda_{2} \ll\left(\frac{\sigma_{n}^{2}}{\sigma_{J}^{2}}\right) \sigma_{S}^{2}
$$

which proves Result 1.

\section{APPENDIX II}

PROOF OF (20)

With

$$
\frac{b^{2}}{a}=\frac{4 \sin ^{2}\left(N x_{0}\right)}{\sin ^{2}\left(x_{0}\right)}\left(\frac{N}{\tan \left(N x_{0}\right)}-\frac{1}{\tan \left(x_{0}\right)}\right)^{2}
$$

deduced from (18), (20) is straightforwardly obtained from a third-order expansion of $\tan \left(N x_{0}\right)$ and $\tan \left(x_{0}\right)$ in $N x_{0}$ and $x_{0}$, respectively, under the assumption that $x_{0} \ll 1$ and $N \gg 1$ with constraint $N x_{0} \ll 1$.

\section{ACKNOWLEDGMENT}

The authors would like to thank Dr. C. Adnet of THALES/ TR6/SR for helpful comments and suggestions on this paper.

\section{REFERENCES}

[1] M. I. Skolnik, Introduction to Radar Systems. New York: McGrawHill, 2002.

[2] H. L. Van Trees, Optimum Array Processing, Part IV of Detection, Estimation and Modulation Theory. New York: Wiley Intersci., 2002.

[3] M. Zatman, "How narrow is narrowband," Inst. Elect. Eng. Proc. Radar, Sonar Navig., vol. 145, pp. 85-91, Apr. 1998.

[4] T. Qin, H. Zhang, and X. Zhang, "Criterion for narrowband beamforming," Inst. Elect. Eng. Electron. Lett., vol. 40, pp. 846-847, Jul. 2004.

[5] H. B. Lee, "Eigenvalues and eigenvectors of covariances matrices for signals closely spaced in frequency," IEEE Trans. Signal Process., vol. 40, no. 10, pp. 2518-2535, Oct. 1992.

[6] J. Hudson, Adaptive Array Principles. London, U.K.: Peter Peregrinus, 1981.

[7] J. T. Mayhan, A. J. Simmon, and W. C. Cummings, "Wideband adaptive antenna nulling using tapped delay-lines," IEEE Trans. Antennas Propag., vol. AP-29, no. 6, pp. 923-936, Nov. 1981.

[8] R. T. Compton, "The bandwidth performance of a two-element adaptive array with tapped delay-line processing," IEEE Trans. Antennas Propag., vol. 36, no. 1, pp. 5-14, Jan. 1988.

[9] L. C. Godara and M. R. S. Jahromi, "Limitations and capabilities of frequency domain broadband constrained beamforming schemes," IEEE Trans. Signal Process., vol. 47, no. 9, pp. 2386-2395, Sep. 1999.

[10] H. Duan, B. P. Ng, and L. M. See, "Broadband beamforming using TDL-form IIR filters," IEEE Trans. Signal Process., vol. 55, no. 3, pp. 990-1002, Mar. 2007.

[11] J. P. Delmas and Y. Meurisse, "Robustness of narrowband DOA algorithms with respect to signal bandwidth," Signal Process., vol. 83, no. 3, pp. 493-510, Feb. 2003.

Marc Oudin was born in Reims, France, in 1979. He received the M.Sc. degree in electrical engineering from the Institut National des Télécommunications (INT), Evry, France, in 2003 and the M.Sc. degree in signal processing from Paris XI University, Orsay, France, in 2004.

Since October 2004, he has been pursuing the Ph.D. degree with the Communications, Image and Information Processing (CITI) Department, INT and Thales Air Systems, Limours, France. His research interests include sensor array processing and radar signal processing.

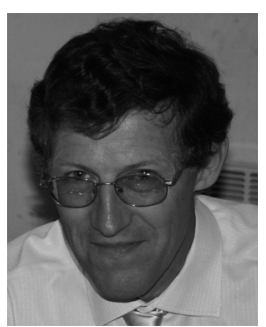

Jean Pierre Delmas (M'00-SM'06) was born in France in 1950. He received the Engineering degree from Ecole Centrale de Lyon, France, in 1973, the Certificat détudes supérieures from the Ecole Nationale Supérieure des Télécommunications, Paris, France, in 1982, and the Habilitation à diriger des recherches (HDR) degree from the University of Paris, Orsay, France, in 2001.

Since 1980, he has been with the Institut National des Télécommunications where he is presently a Professor in the CITI Department and Deputy Director of UMR CNRS 5157 (SAMOVAR) laboratory. His teaching and research interests are in the areas of statistical signal processing with application to communications and antenna array.

Dr. Delmas served as an Associate Editor for the IEEE TRANSACTIONS ON SigNAL PROCESSING (during 2002-2006). 